\title{
LINC02027 / MiR-625-3p /PDLIM5 Axis Inhibits The Proliferation, Migration And Invasion And Promotes Apoptosis of HCC By Inhibiting RAS-MAPK Pathway
}

Jinyi Wang ( $\square$ wywy155725@qq.com )

Jiangsu Province People's Hospital and Nanjing Medical University First Affiliated Hospital: Jiangsu Province Hospital and Nanjing Medical University First Affiliated Hospital https://orcid.org/0000-00015839-0388

\section{Yong Zhu}

Anhui Medical University

Xiaoming Ai

Nanjing BenQ Medical Center

Hong Wan

Anhui Medical University

\section{Wenbo Jia}

Jiangsu Province People's Hospital and Nanjing Medical University First Affiliated Hospital: Jiangsu

Province Hospital and Nanjing Medical University First Affiliated Hospital

Jian Chu

Jiangsu Province People's Hospital and Nanjing Medical University First Affiliated Hospital: Jiangsu

Province Hospital and Nanjing Medical University First Affiliated Hospital

Bin Xu

Jiangsu Province Hospital and Nanjing Medical University First Affiliated Hospital

\section{Xiangxu Kong}

Jiangsu Province People's Hospital and Nanjing Medical University First Affiliated Hospital: Jiangsu

Province Hospital and Nanjing Medical University First Affiliated Hospital

\section{Lianbao Kong}

Jiangsu Province People's Hospital and Nanjing Medical University First Affiliated Hospital: Jiangsu

Province Hospital and Nanjing Medical University First Affiliated Hospital

\section{Research Article}

Keywords: hepatocellular carcinoma, LINC02027, miR-625-3p, PDLIM5, proliferation, migration, invasion, apoptosis, EMT, RAS-MAPK pathway

Posted Date: August 24th, 2021 
DOl: https://doi.org/10.21203/rs.3.rs-820721/v1

License: (c) (1) This work is licensed under a Creative Commons Attribution 4.0 International License. Read Full License 


\section{Abstract}

Background and Aims: Long non-coding RNAs have been shown to promote or inhibit various cancers, acting as competing endogenous RNAs for specific microRNAs. The primary objective of this research was to investigate the underlying mechanism by which the LINC02027/miR-625-3p/PDLIM5 axis affects proliferation, migration, invasion and apoptosis in hepatocellular carcinoma (HCC).

Methods: The level of LINC02027 in HCC tissues and cells and its regulatory effect on the development of HCC were detected. According to the results of database prediction and qRT-PCR, the downstream microRNA and target gene were searched. Finally, HCC cells were transfected with lentivirus and used for cell function assays (in vitro) and subcutaneous tumorigenesis assay in nude mice (in vivo).

Results: Downregulation of LINC02027 was detected in HCC tissues and cell lines. The knockdown or overexpression of LINC02027 increased or reduced the proliferation migration and invasion of HCC cells, and the apoptotic ability of HCC cells decreased or increased, respectively. Mechanistically, INC02027 inhibited epithelial-to-mesenchymal transition (EMT). As a ceRNA, LINC02027 inhibited the malignant ability of HCC by competitively binding to miR-625-3p to regulate the expression of PDLIM5 and the activity of RAS-MAPK pathway.

Conclusion: LINC02027/miR-625-3p/PDLIM5 axis inhibits the EMT and promotes apoptosis of HCC by regulating RAS-MAPK pathway.

\section{Introduction}

Hepatocellular carcinoma is predicted to be the sixth most commonly diagnosed cancer and the fourth leading cause of cancer-related death worldwide ${ }^{1}$. With the continuous development of modern medical care, treatment methods for hepatocellular carcinoma have emerged in an endless stream, such as surgical resection, radiofrequency ablation, liver transplantation, chemotherapy, molecular targeted therapy and immunotherapy, but the five-year survival rate of patients with HCC is still less than $30 \%$ due to its extremely high recurrence and metastasis rates ${ }^{2-4}$. At present, a "ceiling effect" on the treatment of liver cancer is observed, and new diagnostic and treatment methods are urgently needed. Therefore, we must obtain a better understanding of the molecular mechanism underlying the occurrence and progression of HCC.

Long noncoding RNAs (IncRNAs), a class of transcripts over 200 nt in length, were originally thought to be transcriptional noise due to lack of protein-coding ability ${ }^{5,6}$. In recent years, long non-coding RNAs (IncRNAs) has been conspicuous in the study of the molecular mechanism of cancer. Based on accumulating evidence, IncRNAs are involved in many biological processes of HCC, including initiation, growth, metastasis, and therapeutic resistance ${ }^{7-9}$. For instance, LncRNA TRERNA1 promotes sorafenib resistance and cell proliferation in $\mathrm{HCC}^{10}$. LINC-PINT suppresses the aggressiveness of thyroid cancer ${ }^{11}$. LINC00473 functions as an oncogene in colorectal cancer ${ }^{12}$. LncRNAs are not only 
considered biomarkers but also promising therapeutic targets for cancer therapy. LncRNAs, as endogenous RNAs competing for specific microRNAs, regulate the function of microRNAs and the expression of their target genes, thus influencing the occurrence and development of cancer.

In our study, we identified the biological function of LINC002027 in the development of HCC and investigated its potential molecular mechanisms. We performed sequencing analysis on three pairs of $\mathrm{HCC}$ and adjacent tissues, and the sequencing report showed a substantial variation in the expression of LINC02027. At the same time, a bioinformatics website available at ENCORI: The Encyclopedia of RNA Interactomes. (sysu.edu.cn) predicted that LINC02027 was expressed at high levels in adjacent tissues but expressed at low levels in HCC tissues. Our results showed that LINC02027 was downregulated in HCC tissues and cell lines. LINC02027 inhibited cell proliferation, migration and invasion and promoted apoptosis. LINC02027 /miR-625-3p /PDLIM5 axis affected the development of HCC by regulating RAS-MAPK pathway. Our results suggest that LINC02027 is closely associated with HCC progression and may serve as a potential diagnostic biomarker for HCC.

\section{Materials And Methods}

\section{Clinical Tissues}

Sixty paired HCC issues and correlatively nontumor tissues were collected from patients diagnosed with HCC based on the pathological findings after hepatectomy at Jiangsu Province Hospital from 2010 to 2021. No patients received chemotherapy or radiotherapy before surgery. All clinical tissues were immediately snap-frozen in liquid nitrogen after resection and stored at $-80^{\circ} \mathrm{C}$. Qualifications of our study were licensed by the Research Ethics Committee of Nanjing Medical University, China. Written informed consent was obtained from each participant prior to the study.

\section{Cell Culture}

Eight human HCC cell lines (HCCLM3, HepG2, SK-Hep-1, YY8103, Hep-3B, MHCC-97H, and Focus) and one immortalized human liver cell line (LO2) purchased from the Type Culture Collection of the Chinese Academy of Sciences (Shanghai, China) were incubated in high glucose Dulbecco's Modified Eagle Medium (DMEM) (Invitrogen, Thermo Fisher Scientific, Inc., Carlsbad, CA, USA) supplemented with 1\% penicillin/streptomycin (Invitrogen) and 10\% fetal bovine serum (FBS; Gibco, Life Technologies, Carlsbad, CA, USA) at $37 \circ \mathrm{C}$ in humidified atmosphere with $5 \% \mathrm{CO}_{2}$.

\section{Cell Grouping and Transfection}

Lentiviruses overexpressing LINC02027(oe-LINC02027), a small hairpin RNA (sh-RNA) against LINC02027 (sh-LINC02027), miR-625-3p mimic, miR-625-3p inhibitor, PDLIM5 overexpression vector (oePDLIM5), shRNA targeting PDLIM5 (sh-PDLIM5) and the corresponding NC (oe-L-NC, sh-L-NC, miR-mimicNC, miR-inhibitor-NC, oe-P-NC and sh-P-NC,) were packaged by GenePharma (Shanghai, China). Twentyfour hours before transfection, HCC cells were inoculated in six-well plates at a density of $5 \times 10^{4}$ cells per 
well. After reaching $60 \%$ to $70 \%$ confluence, cells were transfected according to the manufacturer's protocol. The sequences are provided in Supporting Table 1.

\section{RNA acquisition and real-time quantitative polymerase chain reaction (RT-qPCR)}

Total RNA was extracted from the collected clinical tissues and cell lines using TRIzol reagent (Invitrogen, CA, USA), quantified with a NanoDrop 2000 spectrophotometer (Nano Drop Technologies, MA, USA) and reverse transcribed into complementary DNA (cDNA) using the Prime Script RT reagent Kit (TaKaRa, Dalian, China). Next, IncRNAs and mRNAs were quantified using SYBR Premix ExTaq II (TaKaRa, Dalian, China) according to the manufacturer's recommendations on an ABI 7900 PCR system (ABI, CA, USA). Differences in fold expression were calculated in Excel (Microsoft Corporation, Redmond, WA, USA) using the $2-\Delta \Delta$ Ct method.

\section{Transwell assays}

Transwell assays were performed by using 8.0- $\mu \mathrm{m}$ Transwell permeable supports (Corning, NY, USA). For cell migration assays, cells $\left(2 \times 10^{4}\right)$ were suspended in $200 \mu \mathrm{l}$ of serum-free medium and seeded into the upper chamber, and $600 \mu \mathrm{l}$ of medium containing $10 \%$ FBS were added to the lower chamber. Forty-eight hours later, the nonmigrated cells were gently removed with cotton swabs. Subsequently, the migrated cells were treated with $4 \%$ paraformaldehyde for 30 minutes, followed by staining with a $0.1 \%$ crystal violet solution. Finally, the migrated cells were imaged using an optical microscope. The number of invasive cells was counted at the magnification of $\times 200$ from 5 random fields and then averaged. The number of cells invaded and migrated in the control group was defined as 1 , and normalized fold changes of invasive and migratory cells in the experimental groups were accordingly calculated. The results were acquired from three independent experiments.

\section{Cell counting kit-8 (CCK-8) analysis}

Transfected cells were seeded into 96 -well plates $\left(1 \times 10^{3} /\right.$ well) with five replicate wells. Ten microliters of CCK8 solution were added to the cells in each well and incubated at $37^{\circ} \mathrm{C}$ with $5 \% \mathrm{CO} 2$ for $2 \mathrm{~h}$. Ultimately, the absorbance at $450 \mathrm{~nm}$ was measured using a microplate reader (Thermo Scientific, USA). The experiment was repeated independently more than three times.

\section{Colony formation assay}

Transfected cells were seeded into 6-well plates ( $5 \times 10^{2}$ cells/well) and cultured with DMEM containing $10 \%$ FBS at $37^{\circ} \mathrm{C}$ in a humidified atmosphere with $5 \% \mathrm{CO} 2$ for 2 weeks. Then, these cells were fixed with $4 \%$ paraformaldehyde and stained with a crystal violet staining solution (Beyotime, Shanghai, China) at room temperature for 15 minutes. Images were obtained with an inverted microscope and colonies were manually counted using ImageJ software.

\section{Scratch wound healing assay}


2. Cell migration was measured using a scratch wound healing assay. Briefly, transfected cells were incubated in 6-well plates and cultured to $80-90 \%$ confluence. Wounds were formed using a plastic scriber by scratching the middle of the plates. A minimum of five randomly chosen areas was measured at different time points $(0$ and $48 \mathrm{~h}$ ) and imaged under an inverted microscope (Olympus, Tokyo, Japan) to determine the distance of cell migration to close the wound area.

\section{5-Ethynyl-20-deoxyuridine (EdU) proliferation assay}

Cell proliferation was measured using an EdU proliferation assay (RiboBio, Nanjing, China). Transfected cells were seeded in 24-well plates at a density of $5 \times 10^{4}$ cells per well and cultured for $24 \mathrm{~h}$. After 5 minutes of washing with PBS, $2 \mathrm{mg} / \mathrm{ml}$ glycine was used to neutralize EdU, and $0.5 \%$ Triton X-100 was used to permeabilize the cells for 20 minutes on a shaking table. Then, DAPI and Apollo staining were performed according to a standard laboratory protocol.

\section{Western Bloting}

Total proteins were extracted from cell lines and tissues using precooled radioimmunoprecipitation assay (RIPA) buffer supplemented with $1 \mathrm{mM}$ phenylmethylsulfonyl fluoride (PMSF), subjected to $10 \%$ sodium dodecyl sulfate-polyacrylamide gel electrophoresis (SDS-PAGE) and transferred onto PVDF membranes (Bio-Rad, CA, USA). Membranes were incubated overnight at $4{ }^{\circ} \mathrm{C}$ with primary antibodies. The protein bands were washed with phosphate-buffered saline Tween-20 (PBST), incubated with a peroxidaseconjugated secondary antibody for $2 \mathrm{~h}$ at $37^{\circ} \mathrm{C}$ and then detected using Super ECL Detection Reagent (Yeasen, Shanghai, China).

\section{Mouse xenograft model}

Ethical approval was obtained from the local ethics committee of the First Affiliated Hospital of Nanjing Medical University, and all animal procedures were performed under the direction of the institution. Male BALB/c nude mice (approximately six weeks) were purchased from the Model Animal Research Center of Nanjing Medical University (Nanjing China) and maintained under clean conditions. Approximately $5 \times 10^{6}$ transfected cells were subcutaneously injected into the dorsal flanks of 4-week-old nude mice. The tumor size was recorded every 3 days, and the mice were sacrificed 4 weeks later. The tumor volumes $\left(\mathrm{mm}^{3}\right)$ were determined using the following formula: volume $=($ shortest diameter) $2 \times$ (longest diameter) $\times 0.5$.

\section{Immunohistochemistry (IHC)}

HCC tissues, adjacent normal tissues and subcutaneous tumors from nude mice were collected in $4 \%$ paraformaldehyde and then embedded in paraffin blocks. The methods for IHC and photographing protocols were conducted and evaluated as previously described. The tumor staining intensity was divided into four gradients: 0 (negative), 1 (weak), 2 (medium), and 3 (strong). The overall score was defined by multiplying the intensity score by the positive rate score. 


\section{Subcellular fractionation analysis}

Cytoplasmic and nuclear RNA were isolated using a PARIS ${ }^{\text {TM }}$ Kit (Ambion, Austin, TX, USA). Transfected cells were suspended in cell fraction buffer and then incubated on ice for $10 \mathrm{~min}$. The upper solution was removed following centrifugation. The nuclear pellet was obtained, while RNA was isolated using cell disruption buffer. Cytoplasmic and nuclear fractions were analyzed using qRT-PCR.

\section{Dual-luciferase reporter assay}

The pGL/Luc-LINC02027-WT/pGL/Luc-LINC02027-MUT and pGL/Luc-PDLIM5-WT/pGL/Luc-PDLIM5MUT vectors (Ambion, Austin, TX, USA) were constructed and then cotransfected with hsa-miR-625-3p mimics, inhibitors or miR-NC into Hep-3B and Focus cells using Lipofectamine 3000 (Invitrogen). After 48

$\mathrm{h}$, the cell lysates were prepared, and the dual-luciferase reporter assay system (Promega, WI, USA) was used to measure dual-luciferase activity.

\section{RNA sequencing}

RNA was extracted from three HCC samples using standard procedures. RNA sequencing was then performed (Ji Guang Gene, Nanjing, China), and the differences were analyzed.

\section{RNA binding protein Immunoprecipitation (RIP) assay}

The RIP assay was performed using a Magna RIP immunoprecipitation kit (Merck Millibo, Billerica, Massachusetts, USA). The supernatant was collected after the hepatoma cells were lysed. Antibody (10 $\mu \mathrm{g}$ ) was added to the supernatant $(10 \mathrm{mg})$ and incubated overnight in a shaker at $4{ }^{\circ} \mathrm{C}$. The next day, Protein $A / G$ magnetic beads $(40 \mu \mathrm{L})$ were added and incubated at $4{ }^{\circ} \mathrm{C}$ for $1 \mathrm{~h}$. The incubation was performed with magnetic beads conjugated to IgG antibody (ab172730; 1:5000; Abcam Inc., Cambridge, MA, USA) or the Ago2 antibody (ab32381; Abcam Inc., Cambridge, MA, USA). The efficacy of RNA immunoprecipitation was verified by qRT-PCR.

\section{Terminal Deoxynucleotide Transferase dUTP Nick End Labeling (TUNEL) assay}

The TUNEL assay was performed using a detection kit (Roche Applied Science, Mannheim, Germany). The heart tissues were rinsed, dehydrated, embedded in paraffin, and cut into $4 \mu \mathrm{m}$ thick slices. Slices were mounted, and the number of TUNEL-positive nuclei was calculated.

\section{Statistical analysis}

Statistical analyses were conducted with GraphPad Prism 5 software (GraphPad Software, San Diego, CA, USA) and SPSS version 18.0 (Chicago, IL, USA). Two-tailed Student's t-test was used to evaluate differences between the two groups. Data are presented as the means \pm standard deviations. A statistically significant difference was defined as $p<0.05\left(^{*}\right)<0.01(* *)<0.001(* * *)$.

\section{Result}




\section{LINC02027 is expressed at low levels in HCC tissues and cells and correlates with a poor outcome}

According to the results of the RNA sequencing assay, LINC02027 was expressed at low levels in three pairs of HCC tissues (Fig. 1 A). Furthermore, we conducted an analysis using the StarBase database, and the result was further confirmed (Fig. S1A). Then, we measured the expression level of LINC02027 in sixty paired HCC tissues and related adjacent nontumor tissues using real-time PCR. LINC02027 expression was significantly downregulated in HCC tumor tissues compared to adjacent nontumor tissues (Fig. 1 B). We generated Kaplan-Meier survival curves to analyze the effect of LINC02027 on the prognosis of patients with HCC and showed that low LINC02027 levels were closely correlated with a higher overall survival rate of patients with HCC (Fig. $1 \mathrm{C}$ ). According to the results of the clinicopathological analysis, LINC02027 expression correlated with tumor diversity, tumor size, Edmondson stage, TNM stage, and microvascular invasion (Table 1). The level of LINC02027 was also detected in HCC cell lines (HCCLM3, HepG2, SK-Hep1, YY8103, Hep-3B, MHCC-97H, and Focus) and compared with the level in the immortalized human liver cell line LO2 (Fig. $1 \mathrm{D}$ ). These results suggested that LINC02027 is downregulated in HCC, which is associated with a poor prognosis and may be a complex factor regulating the occurrence and development of HCC.

\section{Overexpression of LINC02027 inhibits the proliferation, migration, invasion and promotes cell apoptosis of HCC in vitro}

LINC02027 was downregulated in HCC cell lines compared with LO2 cells. Hep-3B and HCCLM3 cells displayed relatively lower expression of LINC02027. Meanwhile, Focus and YY8103 cells expressed LINC02027 at higher levels. Therefore, Hep-3B, YY8103, Focus and HCCLM1 cells were used for the subsequent functional assays. Gain-of-function assays were conducted in Hep-3B and HCCLM3 cells, whereas loss-of-function assays were carried out in Focus and YY8103 cells to investigate the effects of LINC02027 on the occurrence and development of HCC. RT-qPCR results showed that LINC02027 expression was markedly changed in cells transfected with the lentivirus (Fig. S1B-C). We performed CCK-8, colony formation and EdU assays to evaluate the effect of LINC02027 on proliferation. Through these assays, we observed that LINC02027 overexpression notably restrained the proliferation of Hep-3B and HCCLM3 cells. In turn, LINC02027 silencing promoted the proliferation of Focus and YY8103 cells (Fig. 2A, D, F, Fig. S1E-F). Meanwhile, Transwell assays and wound healing assays were performed to evaluate the effects of altered LINC02027 expression on HCC cell migration and invasion.

Overexpressed LINC02027 markedly diminished the number of migrated cells and vice versa (Fig. 2B-C, Fig. S1G). Then, we determined the expression of apoptosis-related genes using real-time PCR. The expression levels of proapoptotic genes (Bax, Caspase-3 and Caspase-9) were obviously increased and that the expression level of the antiapoptotic gene Bcl-2 was dramatically decreased following LINC02027 upregulation (Fig. S1D). The reverse was also true. Similar results were obtained using western blotting (Fig. 2E). After forced expression of LINC02025, the level of the epithelial phenotyperelated marker E-cadherin was decreased, and the levels of $\mathrm{N}$-cadherin and vimentin were increased. After silencing LINC02027, the opposite result was obtained. Based on these results, high LINC02027 
expression inhibited the EMT in HCC cell lines (Fig. 2E). Thus, overexpressed LINC02027 suppresses the growth, migration, and invasive capacity of HCC cells and attenuates cell apoptosis in vitro.

\section{LINC02027 inhibits growth of HCC in vivo}

A subcutaneous tumor model was established to further investigate the carcinogenic effect of LINC02027 in vivo. As shown in the subcutaneous tumor model, LINC02027 overexpression inhibited subcutaneous tumor growth in nude mice, while the downregulation of LINC02027 promoted tumor growth in nude mice, and the results were reflected in tumor weight and volume (Fig. 3A-B, D). HE, Ki67, Ecadherin and Vimentin staining of xenograft tumor tissues showed that Ki67 and Vimentin expression was significantly reduced and E-cadherin expression was increased in the YY8103-LINC02027 group, whereas the opposite results were obtained in the HCCLM3-shLINC02027 group (Fig. 3C). These data suggested that LINC02027 inhibits HCC growth and the EMT in vivo.

\section{LINC02027 directly binds with miR-625-3p in HCC}

We first performed a subcellular fractionation assay to detect the subcellular distribution of LINC02027 and investigate the mechanism of LINC02027 in HCC, and we confirmed that LINC02027 was mainly distributed in the cytoplasm of cancer cells (Fig. 4A). Thus, LINC02027 might function as a ceRNA, a common mechanism of posttranscriptional regulation mediated by IncRNAs. Therefore, we hypothesized that LINC02027 might act as a ceRNA to restrain mRNA expression by chelating a miRNAs. We examined the StarBase database and found that five miRNAs have complementary sites with LINC02027. Next, the qRT-PCR analysis showed that only miR-625-3p changed correspondingly with the expression level of LINC02027 (Fig. S2A). Subsequently, we determined the expression of miR-625-3p in 60 pairs of HCC tissues and related adjacent nontumor tissues using qRT-PCR. As expected, its expression was overtly upregulated in HCC tissues and cell lines (Fig. 4B, D). Spearman's correlation analysis revealed that LINC02027 expression was negatively associated with miR-625-3p (Fig. 4C). A dual-luciferase reporter assay was conducted to validate that LINC02027 directly binds to miR-625-3p and confirm this hypothesis (Fig. 4E). The wild-type (WT) or mutant (MUT) LINC02027 3' UTR was inserted into a reporter vector. The dual-luciferase reporter gene assay results showed that the relative luciferase activities in Hep-3B and Focus cells cotransfected with miR-625-3p and wild-type LINC02027 (Wt-LINC02027) were obviously decreased compared with cells transfected with the mutant (Mut-LINC02027). Conversely, the miR-625-3p inhibitor inhibited the relative luciferase activities (Fig. 4F). Therefore, we conceived that LINC02027 directly binds to miR-625-3p in HCC.

\section{MiR-625-3p promotes the proliferation, migration, and invasion of HCC}

We hypothesized that LINC02027 functions by directly binding to miR-625-3p. We transfected miR-625-3p inhibitors and mimics into Hep3B and Focus cells, respectively (Fig. S2B). Based on the results of CCK-8, colony formation, Transwell and wound healing assays, we concluded that miR-625-3p promotes the proliferation, migration, and invasion of HCC cells (Fig. S2C-F). 


\section{LINC02027 inhibits proliferationDinvasion and migration and promotes apoptosis in the HCC by sponging miR-625-3p}

Hep-3B cells were transfected with oe-L-NC+ miR-mimic-NC, oe-LINC02027+ miR-625-3p mimic-NC and oe-LINC02027+ miR mimic to further explore the relationship between LINC02027 and miR-625-3p. Similarly, sh-L-NC+ miR-inhibitor-NC, sh-LINC02027+ miR- inhibitor-NC and sh-LINC02027+ miR-625-3p inhibitor were transfected into Focus cells. The transfection efficiency was verified using RT-PCR (Fig. S3A). A series of rescue experiments were conducted to confirm that LINC02027 is the upstream regulator of miR-625-3p. By repeating the experiments described above, we found that miR-625-3p maintained the malignant phenotype of HCC cells (Fig. 5A, C-E, Fig. S3 B, D-F). Western blotting analysis showed that miR-625-3p rescued the inhibitory effect of LINC02027 on the EMT and the stimulation of apoptosis in HCC cell lines (Fig. 5B, Fig. S3 C). We established a model of xenograft tumor formation in vivo to further investigate the relationship between LINC02027 and miR-625-3p and their respective effects on the proliferation ability of HCC cells. Due to the low tumorigenicity of the Hep-3B and Focus cell lines, we used the YY8103 cell line for validation in vivo. The transfected YY8103 cells were injected subcutaneously into nude mice. The volume and weight of subcutaneous tumors in different groups were recorded in real time (Fig. 5F-G). Both HE staining and immunohistochemical staining of subcutaneous tumors showed that LINC02027 inhibited the proliferation of HCC cells, while miR-625-3p exerted the opposite effect (Fig. 5H). Through the aforementioned experiments, we confirmed that LINC02027 inhibits the malignancy of HCC cells by regulating miR-625-3p.

\section{LINC02027 promotes HCC Cell Proliferation, Migration, and Invasion by Targeting miR-625-3p/PDLIM5}

Based on publicly available databases, including miRDB, mirDIP, TargetScan and miRWalk, we identified seven potential downstream target genes (Fig. 6A). According to the prediction of the StarBase database, only PDLIM5 was significantly differentially expressed in HCC, and the trend was consistent with our initial conjecture (Fig. S4A). In addition, the prediction from the Kaplan-Meier Plotter database indicated that PDLIM5 affected the overall survival and disease-free survival of individuals with HCC (Fig. S4B). The expression level of PDLIM5 in HCC tissues and cells was verified using qRT-PCR, and the results were consistent with the prediction provided by the database (Fig. 6B, E). Spearman's correlation analysis revealed that PDLIM5 expression was negatively associated with miR-625-3p expression and positively correlated with LINC02027 expression (Fig. 6C). Western blotting analysis revealed lower levels of the PDLIM5 protein in HCC tissues and cells than in related adjacent nontumor tissues (Fig. 6D). Then, we also verified the binding between miR-625-3p and PDLIM5 through a dual-luciferase reporter assay (Fig. $6 G$ ). Lentiviruses carrying a short hairpin RNA (shRNA) or negative control (shNC) were transfected into Hep-3B or Focus cell lines. The luciferase activity of the miR/PDLIM5-WT system was dramatically decreased in the miR-625-3p mimic group, but it was increased in the miR-625-3p inhibitor group, with no obvious difference in luciferase activity compared with the miR/PDLIM5-Mut system (Fig. 6F). Moreover, RIP test results showed that LINC02027, miR-625-3p and PDLIM5 were significantly enriched in the antiAGO2 group compared with the (anti-lgG) negative control group (Fig. $6 \mathrm{H}$ ). Therefore, we speculated that 
LINC02027 affects the occurrence and development of HCC by competitively binding to miR-625-3p and thereby affecting the downstream target gene PDLIM5.

In the cells described above, PDLIM5 expression was significantly changed after transfection with either the mimic or inhibitor but not after transfection with miR-mimic-NC or miR-inhibitor-NC (Fig. S4C). The results of the CCK-8 assay, scratch wound healing test, Transwell assay and EdU assay provided evidence that cell viability, migration and invasion were apparently increased by the transfection of oe-PDLIM5 or miR-625-3p inhibitor but were dramatically decreased by the transfection of sh-PDLIM5 or miR-625-3p mimic (Fig. 7A, C-E, G, Fig. S4D-G, Fig. S5A). In summary, miR-625-3p targets PDLIM5, facilitates the proliferation, migration and invasion abilities and suppresses the apoptosis of HCC cells.

This hypothesis was confirmed not only in vitro experiments but also in vivo experiments. The transfected cells were injected subcutaneously into nude mice, and the tumor size was observed and recorded regularly. Then, the subcutaneously obtained tumors were subjected to H\&E staining and immunohistochemistry (Fig. 8A-C). Finally, TUNEL assay were performed to evaluate cell apoptosis. (Fig. 8D). These results were consistent with our expectations. Taken together, LINC02027 regulates PDLIM5 by binding to miR-625-3p, which further inhibits the development of HCC and stimulates apoptosis.

\section{LINC02027 / miR-625-3p /PDLIM5 axis can affect the proliferation, migration, invasion and apoptosis of HCC by regulating RAS-MAPK pathway}

We used the StarBase database to analyze the potential downstream targets of PDLIM5 and found that the expression of RASA1 (RAS P21 protein activator 1), which inhibits the RAS-MAPK pathway, was positively correlated with PDLIM5 expression (Fig. S5C). We hypothesized that LINC02027 affects the RAS-MAPK pathway by altering the expression of PDLIM5. Then, western blotting was used to detect the activity of the RAS-MAPK pathway. Compared with the control group, the levels of phosphorylated Raf, MEK and ERK proteins in LINC02027-overexpressing Hep-3B cells were significantly decreased, while the levels of the RASA1 protein were significantly decreased. Knockdown of PDLIM5 reversed the aforementioned changes in protein expression (Fig. 7B, Fig. S5B). These findings indicated that the regulation of the RAS-MAPK pathway by the LINC02027/miR-625-3p/PDLIM5 axis affects the proliferation, migration, invasion and apoptosis of HCC.

\section{Discussion}

In recent years, remarkable progress and development have been achieved in a series of treatment methods for HCC, but traditional treatment methods have exhibited a "ceiling effect", increasing the difficulty of further significantly improving the efficacy. Therefore, a better understanding of the potential mechanism of the occurrence and development of HCC is urgently needed ${ }^{13}$. LncRNAs have recently emerged as promising participants in the regulation of gene expression through novel mechanisms compared to mRNA-encoding genes ${ }^{14}$. According to previous studies, the dysregulation of IncRNAs is 
associated with the occurrence and progression of malignancies, suggesting their clinical potential as biomarkers for diagnosis and prognosis, as well as therapeutic targets ${ }^{15-17}$.

We observed a decrease in LINC02027 expression in HCC and predicted that a low level of LINC02027 was associated with a poor prognosis for patients with HCC. LINC02027 suppresses the proliferation, migration, and invasion and promotes the apoptosis of HCC. Little is known about the functional role and clinical significance of LINC02027 in HCC. First, LINC02027 was screened by searching the sequencing analysis report and GEPIA and StarBase databases. We further validated this finding in a group of HCC and related adjacent nontumor tissues, as well as in HCC cell lines and normal liver cell lines. We then knocked down or overexpressed LINC02027 in HCC cell lines by lentivirus transfection. In the Focus and HCCLM3 cell lines, the downregulation of LINC02027 promoted proliferation, migration, and invasion and inhibited apoptosis. However, in the Hep-3B and YY8103 cell lines, the opposite effect was detected when LINC02027 was overexpressed. The knockout and overexpression of LINC00978 also influenced the growth of HCC in vivo. Both in vivo and in vitro studies have shown that LINC00978 is critical for HCC progression. Our study indicated that LINC02027 may serve as a new prognostic biomarker and a potential therapeutic target for HCC.

The function of IncRNAs is determined by their subcellular fate ${ }^{18}$. LncRNAs localized in the cytoplasm influence gene regulation by acting as decoys for miRNAs and interfering with the interaction of miRNAs and their target mRNAs ${ }^{19-21}$. The IncRNA-miRNA-mRNA interaction network has been proven to be an important mechanism underlying the occurrence and development of different types of cancer, including HCC $^{22-24}$. For example, the IncRNA SNHG6 promotes the G1/S-phase transition in hepatocellular carcinoma by impairing miR-204-5p-mediated inhibition of E2F1 ${ }^{25}$. miR-625-3p regulates oxaliplatin resistance by targeting MAP2K6-p38 signaling in human colorectal adenocarcinoma cells ${ }^{26}$. Our findings suggest that LINC02027 may be involved in the process of HCC by acting as a ceRNA for miR-625-3p, resulting in the downregulation of PDLIM5.

As an actin connector protein, PDLIM5 is not only involved in cytoskeletal tissue, cellular processes and organ development but is also presumed to be involved in the genesis and development of tumors ${ }^{27}$. PDLIM5 has been reported to play an important role in a variety of cancers, including papillary thyroid cancer, prostate cancer and non-small cell lung cancer ${ }^{28-30}$. Based on publicly available databases, we found that PDLIM5 may be a potential downstream target of LINC02027. Simultaneously, dual-luciferase reporter and rescue analysis were performed to verify the accuracy of the prediction. LINC02027 acted as a bait for miR-625a-3p and competitively bound to miR-625a-3p, thereby altering the expression of PDLIM5 in HCC.

The mitogen-activated protein kinase (MAPK) cascade is a key signaling pathway regulating cell proliferation, differentiation, apoptosis and other processes, and the Ras/Raf/MAPK/ERK pathway is the most important signaling cascade in the pathway ${ }^{31}$. The development of various human tumors, such as ovarian, colon, breast and endometrial cancers, is related to the activation of the RAS/MAPK 
pathway ${ }^{32,33}$. The basic signal transduction steps follow the three-stage MAPK enzyme cascade, which consists of the upstream activated proteins MAP3K, MAP2K and MAPK. In the ERK pathway, Ras

functions as an upstream activating protein to form the RAS-Raf-MEK-ERK pathway ${ }^{34}$. Western blotting analysis showed that low expression of LINC02027 and PDLIM5 increased the levels of proteins in the RAS-Raf-MEK-ERK pathway. Therefore, we postulate that LINC02027 inhibits the activation of the RASRaf-MEK-ERK pathway by regulating PDLIM5 expression, thus inhibiting the proliferation, migration and invasion of liver cancer and promoting apoptosis.

\section{Conclusions}

In summary, the present study revealed the role of the LINC02027/miR-625-3p/PDLIM5/RAS/MAPK axis in the development of HCC. The upregulation of LINC02027 inhibited the proliferation, migration and invasion of HCC cells and promote apoptosis by upregulating PDLIM5 and inhibiting the RAS-Raf-MEKERK pathway. Therefore, LINC02027 represents a potentially useful cancer inhibitor and a potential therapeutic target for HCC. However, more efforts are needed in future studies to elucidate the specific mechanisms by which LINC02027 affects the occurrence and progression of HCC.

\section{List Of Abbreviations}

HCC: hepatocellular carcinoma; EMT: epithelial-to-mesenchymal transition; DMEM: Dulbecco's modified Eagle's medium; FBS: fetal bovine serum; RT-qPCR: real-time quantitative polymerase chain reaction; IHC: Immunohistochemistry; CCK-8: Cell Counting Kit-8; TNM: tumor-node-metastasis; HE: Hematoxylin and eosin;

\section{Declarations}

\section{Ethics approval and consent to participate}

This study was permitted by the local Ethics Committee from The First Affiliated Hospital of Nanjing Medical University (Nanjing, China).

\section{Consent for publication}

All authors agree on publication of the present manuscript.

\section{Availability of data and materials}

All data that generated during this study are available from the corresponding author upon reasonable request.

\section{Competing interests}

The authors declare no conflict of interest. 


\section{Funding}

This work was supported by grants from the Natural Science Foundation of China (81871260); Basic and Clinical Cooperative Research Promotion Program of Anhui Medical University (No.2019xkjT029); Nanjing Health science and Technology Development Special fund Project (NO.YKK20218);

\section{Authors' contributions}

JW, $Y Z$ and $X A$ conducted the experiments and wrote this manuscript. HW, WJ, JW, BX and XK conducted parts of the experiments. LK analyzed the data. LK conceived and supervised this study. All authors have read and approved the final manuscript.

\section{Acknowledgements}

Not applicable.

\section{References}

1 Bray, F., Ferlay, J., Soerjomataram, I., Siegel, R. L., Torre, L. A. \& Jemal, A. Global cancer statistics 2018: GLOBOCAN estimates of incidence and mortality worldwide for 36 cancers in 185 countries. $C A$ Cancer J Clin 68, 394-424, doi:10.3322/caac.21492 (2018).

2 Goh, G. B., Chang, P. E. \& Tan, C. K. Changing epidemiology of hepatocellular carcinoma in Asia. Best Pract Res Clin Gastroentero/ 29, 919-928, doi:10.1016/j.bpg.2015.09.007 (2015).

3 Xu, X., Gu, J., Ding, X., Ge, G., Zang, X., Ji, R., Shao, M., Mao, Z., Zhang, Y., Zhang, J., Mao, F., Qian, H., Xu, W., Cai, H., Wang, F. \& Zhang, X. LINC00978 promotes the progression of hepatocellular carcinoma by regulating EZH2-mediated silencing of p21 and E-cadherin expression. Cell Death Dis 10, 752, doi:10.1038/s41419-019-1990-6 (2019).

4 Galun, D., Srdic-Rajic, T., Bogdanovic, A., Loncar, Z. \& Zuvela, M. Targeted therapy and personalized medicine in hepatocellular carcinoma: drug resistance, mechanisms, and treatment strategies. $J$ Hepatocell Carcinoma 4, 93-103, doi:10.2147/JHC.S106529 (2017).

5 Rajagopal, T., Talluri, S., Akshaya, R. L. \& Dunna, N. R. HOTAIR LncRNA: A novel oncogenic propellant in human cancer. Clin Chim Acta 503, 1-18, doi:10.1016/j.cca.2019.12.028 (2020).

6 Zhang, J., Ding, T. \& Zhang, H. Insight Into Chromatin-Enriched RNA: A Key Chromatin Regulator in Tumors. Front Cell Dev Bio/ 9, 649605, doi:10.3389/fcell.2021.649605 (2021).

7 Peng, L., Jiang, B., Yuan, X., Qiu, Y., Peng, J., Huang, Y., Zhang, C., Zhang, Y., Lin, Z., Li, J., Yao, W., Deng, W., Zhang, Y., Meng, M., Pan, X., Li, C., Yin, D., Bi, X., Li, G. \& Lin, D. C. Super-Enhancer-Associated 
Long Noncoding RNA HCCL5 Is Activated by ZEB1 and Promotes the Malignancy of Hepatocellular Carcinoma. Cancer Res 79, 572-584, doi:10.1158/0008-5472.CAN-18-0367 (2019).

8 Wu, J., Zhu, P., Lu, T., Du, Y., Wang, Y., He, L., Ye, B., Liu, B., Yang, L., Wang, J., Gu, Y., Lan, J., Hao, Y., He, L. \& Fan, Z. The long non-coding RNA LncHDAC2 drives the self-renewal of liver cancer stem cells via activation of Hedgehog signaling. J Hepatol 70, 918-929, doi:10.1016/j.jhep.2018.12.015 (2019).

9 Rothschild, G., Zhang, W., Lim, J., Giri, P. K., Laffleur, B., Chen, Y., Fang, M., Chen, Y., Nair, L., Liu, Z. P., Deng, H., Hammarstrom, L., Wang, J. \& Basu, U. Noncoding RNA transcription alters chromosomal topology to promote isotype-specific class switch recombination. Sci Immuno/ 5, doi:10.1126/sciimmunol.aay5864 (2020).

10 Song, W., Zheng, C., Liu, M., Xu, Y., Qian, Y., Zhang, Z., Su, H., Li, X., Wu, H., Gong, P., Li, Y. \& Fan, H. LncRNA TRERNA1 upregulation mediated by $\mathrm{HBx}$ promotes sorafenib resistance and cell proliferation in HCC via targeting NRAS by sponging miR-22-3p. Mol Ther, doi:10.1016/j.ymthe.2021.04.011 (2021).

11 Jia, M., Li, Z., Pan, M., Tao, M., Wang, J. \& Lu, X. LINC-PINT Suppresses the Aggressiveness of Thyroid Cancer by Downregulating miR-767-5p to Induce TET2 Expression. Mol Ther Nucleic Acids 22, 319-328, doi:10.1016/j.omtn.2020.05.033 (2020).

12 Wang, L., Zhang, X., Sheng, L., Qiu, C. \& Luo, R. LINC00473 promotes the Taxol resistance via miR15a in colorectal cancer. Biosci Rep 38, doi:10.1042/BSR20180790 (2018).

13 Lurje, I., Czigany, Z., Bednarsch, J., Roderburg, C., Isfort, P., Neumann, U. P. \& Lurje, G. Treatment Strategies for Hepatocellular Carcinoma (-) a Multidisciplinary Approach. Int J Mol Sci 20, doi:10.3390/ijms20061465 (2019).

14 Xie, S. C., Zhang, J. Q., Jiang, X. L., Hua, Y. Y., Xie, S. W., Qin, Y. A. \& Yang, Y. J. LncRNA CRNDE facilitates epigenetic suppression of CELF2 and LATS2 to promote proliferation, migration and chemoresistance in hepatocellular carcinoma. Cell Death Dis 11, 676, doi:10.1038/s41419-020-02853-8 (2020).

15 Gupta, R. A., Shah, N., Wang, K. C., Kim, J., Horlings, H. M., Wong, D. J., Tsai, M. C., Hung, T., Argani, P., Rinn, J. L., Wang, Y., Brzoska, P., Kong, B., Li, R., West, R. B., van de Vijver, M. J., Sukumar, S. \& Chang, H. Y. Long non-coding RNA HOTAIR reprograms chromatin state to promote cancer metastasis. Nature 464, 1071-1076, doi:10.1038/nature08975 (2010).

16 Kim, Y. A., Park, K. K. \& Lee, S. J. LncRNAs Act as a Link between Chronic Liver Disease and Hepatocellular Carcinoma. Int J Mol Sci 21, doi:10.3390/ijms21082883 (2020).

17 Ma, M., Xu, H., Liu, G., Wu, J., Li, C., Wang, X., Zhang, S., Xu, H., Ju, S., Cheng, W., Dai, L., Wei, Y., Tian, Y. \& Fu, X. Metabolism-induced tumor activator 1 (MITA1), an Energy Stress-Inducible Long Noncoding 
RNA, Promotes Hepatocellular Carcinoma Metastasis. Hepatology 70, 215-230, doi:10.1002/hep.30602 (2019).

18 Chen, L. L. Linking Long Noncoding RNA Localization and Function. Trends Biochem Sci 41, 761772, doi:10.1016/j.tibs.2016.07.003 (2016).

19 Fasolo, F., Patrucco, L., Volpe, M., Bon, C., Peano, C., Mignone, F., Carninci, P., Persichetti, F., Santoro, C., Zucchelli, S., Sblattero, D., Sanges, R., Cotella, D. \& Gustincich, S. The RNA-binding protein ILF3 binds to transposable element sequences in SINEUP IncRNAs. FASEB J 33, 13572-13589, doi:10.1096/fj.201901618RR (2019).

20 Sas-Chen, A., Srivastava, S. \& Yarden, Y. The short and the long: non-coding RNAs and growth factors in cancer progression. Biochem Soc Trans 45, 51-64, doi:10.1042/BST20160131 (2017).

21 Ye, J. R., Liu, L. \& Zheng, F. Long Noncoding RNA Bladder Cancer Associated Transcript 1 Promotes the Proliferation, Migration, and Invasion of Nonsmall Cell Lung Cancer Through Sponging miR-144. DNA Cell Bio/ 36, 845-852, doi:10.1089/dna.2017.3854 (2017).

22 Zhao, J., Xu, J., Wang, W., Zhao, H., Liu, H., Liu, X., Liu, J., Sun, Y., Dunaif, A., Du, Y. \& Chen, Z. J. Long non-coding RNA LINC-01572:28 inhibits granulosa cell growth via a decrease in p27 (Kip1) degradation in patients with polycystic ovary syndrome. EBioMedicine 36, 526-538, doi:10.1016/j.ebiom.2018.09.043 (2018).

23 Liu, Q., Zhang, W., Wu, Z., Liu, H., Hu, H., Shi, H., Li, S. \& Zhang, X. Construction of a circular RNAmicroRNA-messengerRNA regulatory network in stomach adenocarcinoma. J Cell Biochem 121, 13171331, doi:10.1002/jcb.29368 (2020).

24 Lyu, L., Xiang, W., Zhu, J. Y., Huang, T., Yuan, J. D. \& Zhang, C. H. Integrative analysis of the IncRNAassociated ceRNA network reveals IncRNAs as potential prognostic biomarkers in human muscleinvasive bladder cancer. Cancer Manag Res 11, 6061-6077, doi:10.2147/CMAR.S207336 (2019).

25 Chen, K., Hou, Y., Liao, R., Li, Y., Yang, H. \& Gong, J. LncRNA SNHG6 promotes G1/S-phase transition in hepatocellular carcinoma by impairing miR-204-5p-mediated inhibition of E2F1. Oncogene 40, 32173230, doi:10.1038/s41388-021-01671-2 (2021).

26 Rasmussen, M. H., Lyskjaer, I., Jersie-Christensen, R. R., Tarpgaard, L. S., Primdal-Bengtson, B., Nielsen, M. M., Pedersen, J. S., Hansen, T. P., Hansen, F., Olsen, J. V., Pfeiffer, P., Orntoft, T. F. \& Andersen, C. L. miR-625-3p regulates oxaliplatin resistance by targeting MAP2K6-p38 signalling in human colorectal adenocarcinoma cells. Nat Commun 7, 12436, doi:10.1038/ncomms12436 (2016).

27 Huang, X., Qu, R., Ouyang, J., Zhong, S. \& Dai, J. An Overview of the Cytoskeleton-Associated Role of PDLIM5. Front Physio/ 11, 975, doi:10.3389/fphys.2020.00975 (2020). 
28 Shi, Y., Wang, X., Xu, Z., He, Y., Guo, C., He, L., Huan, C., Cai, C., Huang, J., Zhang, J., Li, Y., Zeng, C., Zhang, X., Wang, L., Ke, Y. \& Cheng, H. PDLIM5 inhibits STUB1-mediated degradation of SMAD3 and promotes the migration and invasion of lung cancer cells. J Biol Chem 295, 13798-13811, doi:10.1074/jbc.RA120.014976 (2020).

29 Wei, X., Zhang, Y., Yu, S., Li, S., Jiang, W., Zhu, Y., Xu, Y., Yang, C., Tian, G., Mi, J., Bergquist, J., Zhao, M. \& Song, F. PDLIM5 identified by label-free quantitative proteomics as a potential novel biomarker of papillary thyroid carcinoma. Biochem Biophys Res Commun 499, 338-344, doi:10.1016/j.bbrc.2018.03.159 (2018).

30 Liu, X., Chen, L., Huang, H., Lv, J. M., Chen, M., Qu, F. J., Pan, X. W., Li, L., Yin, L., Cui, X. G., Gao, Y. \& $\mathrm{Xu}, \mathrm{D}$. F. High expression of PDLIM5 facilitates cell tumorigenesis and migration by maintaining AMPK activation in prostate cancer. Oncotarget 8, 98117-98134, doi:10.18632/oncotarget.20981 (2017).

31 Braeuning, A. \& Schwarz, M. Regulation of expression of drug-metabolizing enzymes by oncogenic signaling pathways in liver tumors: a review. Acta Pharm Sin B 10, 113-122, doi:10.1016/j.apsb.2019.06.013 (2020).

32 Rao, A. \& Herr, D. R. G protein-coupled receptor GPR19 regulates E-cadherin expression and invasion of breast cancer cells. Biochim Biophys Acta Mol Cell Res 1864, 1318-1327, doi:10.1016/j.bbamcr.2017.05.001 (2017).

33 Bhartiya, D. \& Singh, J. FSH-FSHR3-stem cells in ovary surface epithelium: basis for adult ovarian biology, failure, aging, and cancer. Reproduction 149, R35-48, doi:10.1530/REP-14-0220 (2015).

34 Yang, S. \& Liu, G. Targeting the Ras/Raf/MEK/ERK pathway in hepatocellular carcinoma. Oncol Lett 13, 1041-1047, doi:10.3892/ol.2017.5557 (2017).

\section{Figures}


A

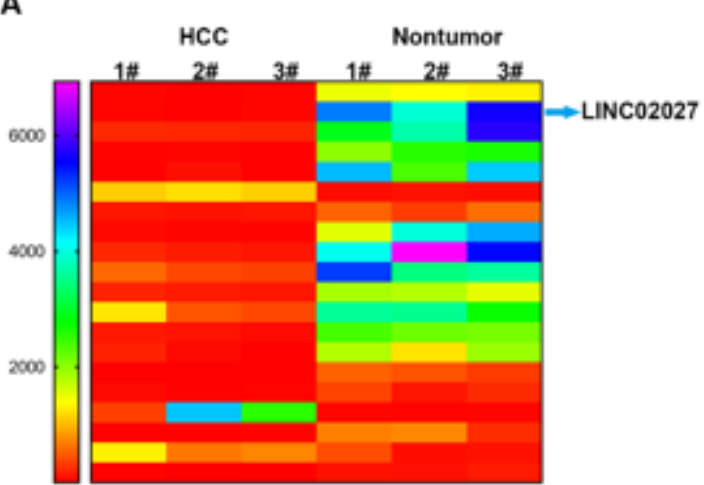

C

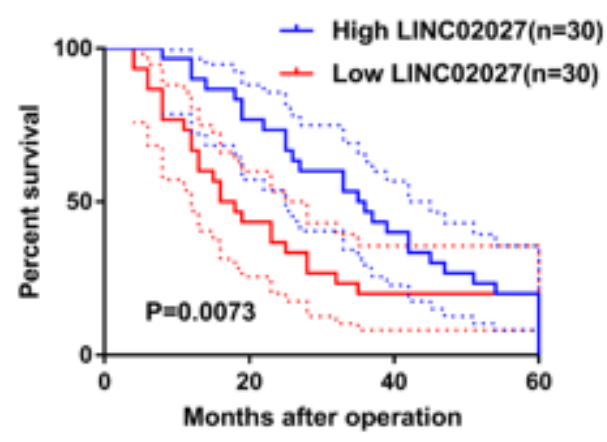

B

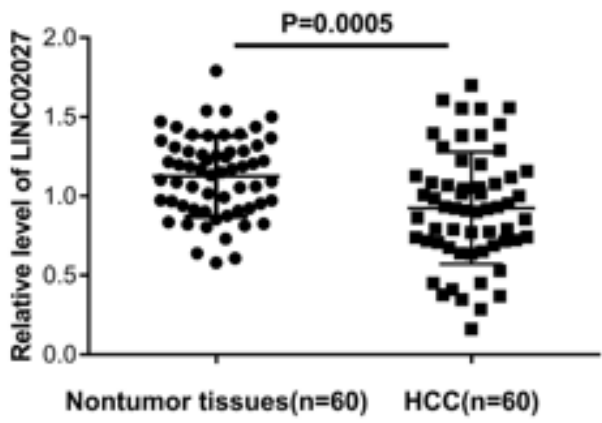

D

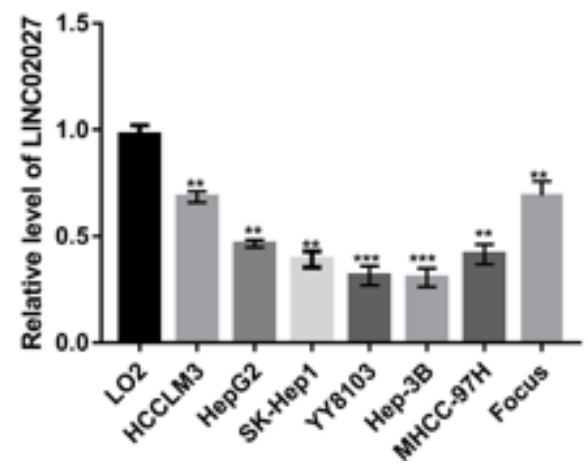

\section{Figure 1}

LINC02027 is down-regulated in HCC and has correlation with clinical prognosis. A. Heat map of the IncRNA sequencing assay. B. The expression of LINC02027 was analyzed in 60 pairs of HCC tissues and related nontumor tissues. C. Kaplan-Meier analyses of the correlation between LINC02027 RNA levels and the overall survival in 60 patients with HCC. Patients were stratified for the analysis by log2Foldchange $(T / N)$, log2Foldchange $(T / N) \geq 1$, labled as high LINC02027; otherwise labled as low LINC02027. D. The expression of LINC02027 in HCC cell lines and immortalized human hepatocyte LO2. The $p$ values represent comparisons between groups $\left({ }^{\star} p<0.05,{ }^{\star \star} p<0.01,{ }^{\star \star \star} p<0.001\right)$. 


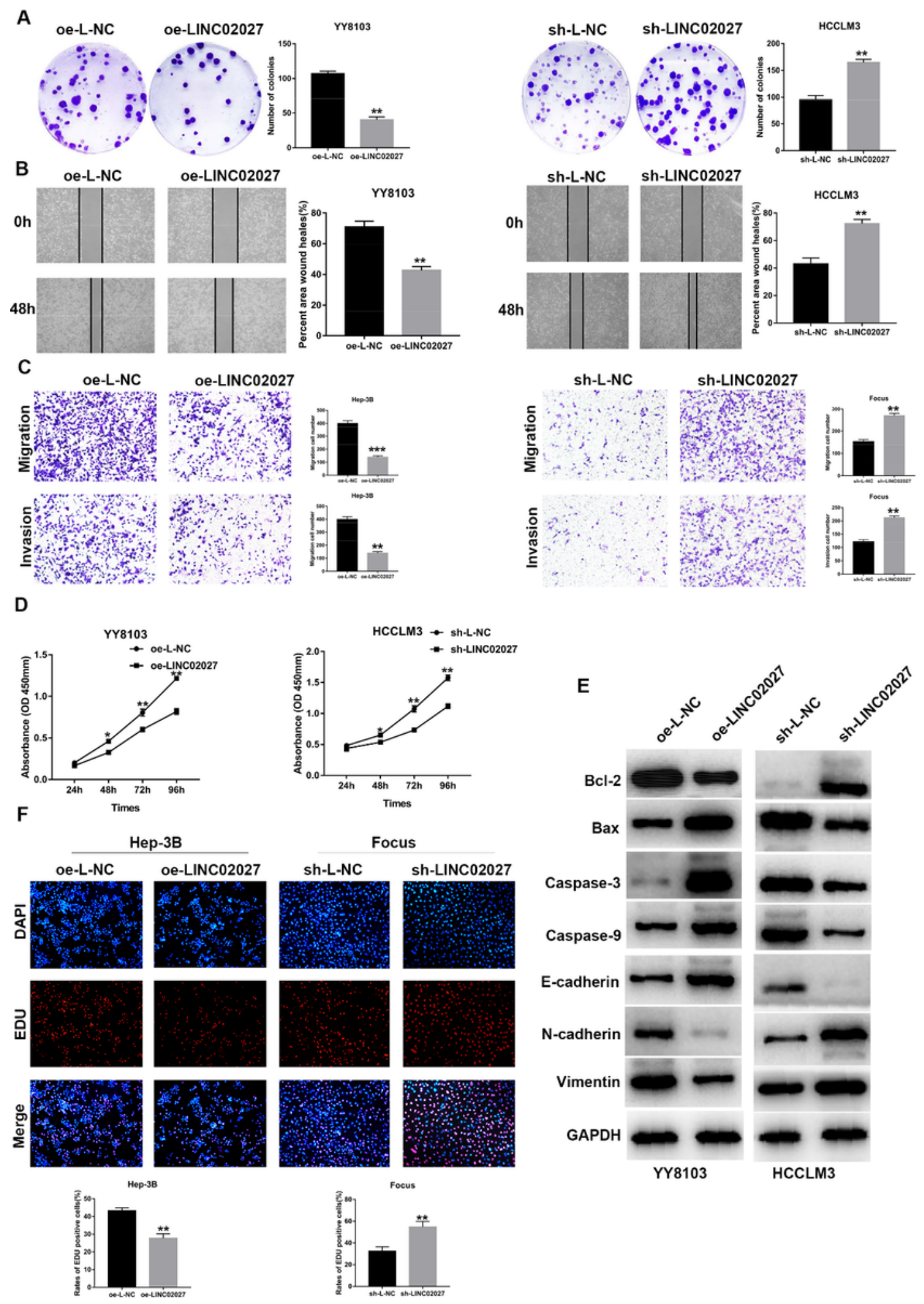

Figure 2

The expression of LINC02027 is inversely correlated with the proliferation, migration and invasion abilities of HCC cell lines. A. Representative images of colony formation assay of YY8103 and HCCLM3 cells with different treatments. B. Wound healing assay suggested that the up-regulation and downregulation of LINC02027 changed motility in HCC cell lines. C. Transwell assays showed that the transfected cells changed invasion and migration ability. D. Growth curves based on CCK-8 assay in 
transfected cells. E. Apoptosis-related and EMT-associated proteins were detected by western blot analysis. F. The results of the EdU assay showed that up-regulation and downregulation of LINC02027 in Hep-3B and Focus cells inhibits cell proliferation.

A
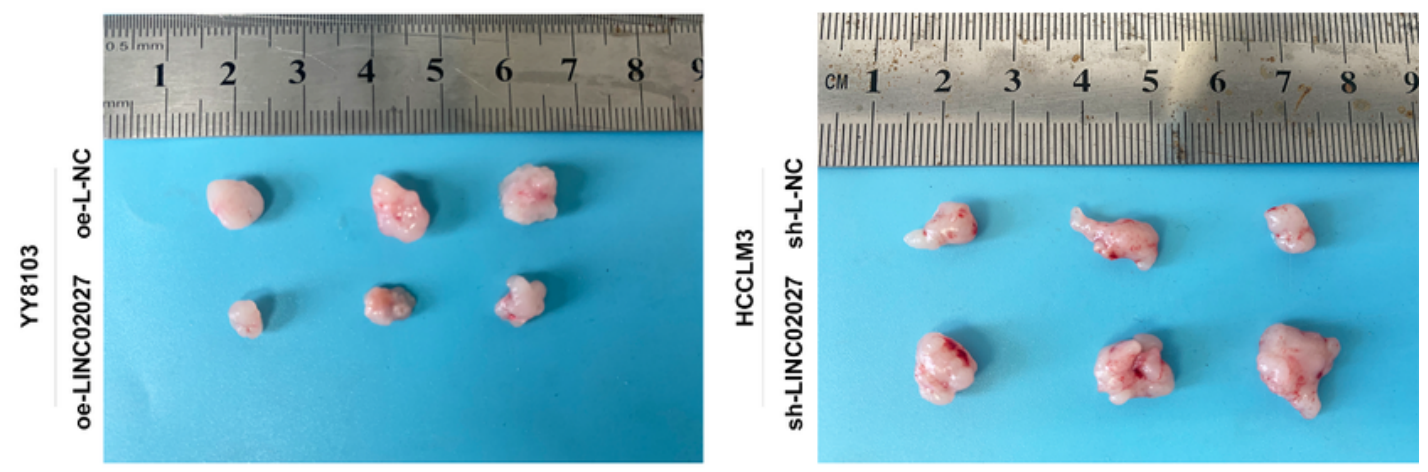

B

C
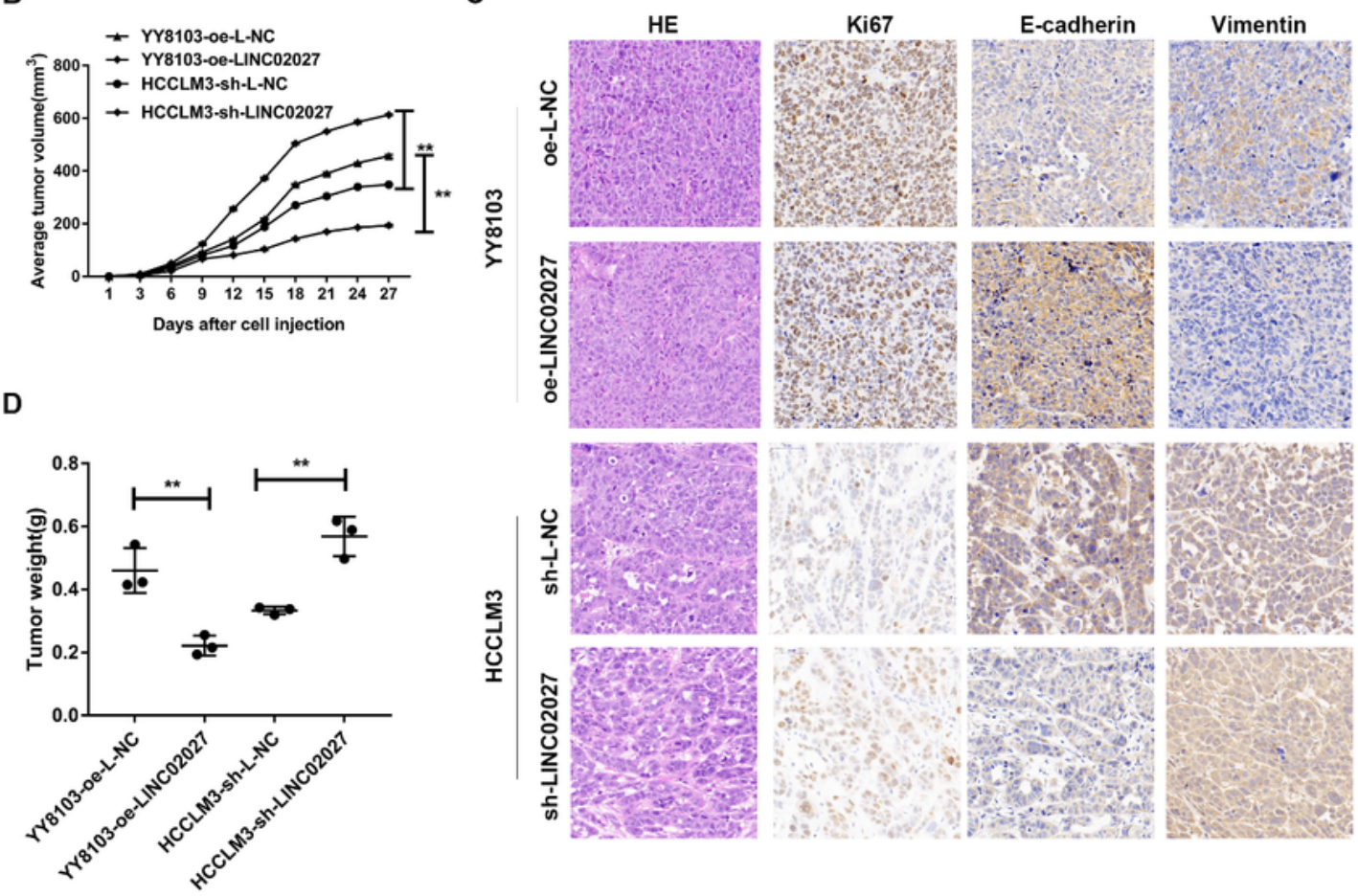

Figure 3

In vivo experiments, the inhibitory effect of LINC02027 on HCC. A. Photograph of tumors obtained from nude mice. B. Tumor volume growth curve. C. Representative images of HE staining and 
Immunohistochemical detection of Ki67, E-cadherin and Vimentin protein levels in four groups of subcutaneous tumor tissues $(x 400)$. D. Tumor weight. The $p$ values represent comparisons between groups $\left({ }^{*} p<0.05,{ }^{* \star} p<0.01,{ }^{* \star *} p<0.001\right)$.In vivo experiments, the inhibitory effect of LINC02027 on HCC. A. Photograph of tumors obtained from nude mice. B. Tumor volume growth curve. C.

Representative images of HE staining and Immunohistochemical detection of Ki67, E-cadherin and Vimentin protein levels in four groups of subcutaneous tumor tissues $(x 400)$. D. Tumor weight. The $p$ values represent comparisons between groups $\left({ }^{*} p<0.05\right.$, $\left.{ }^{*} p<0.01,{ }^{* \star *} p<0.001\right)$.

A

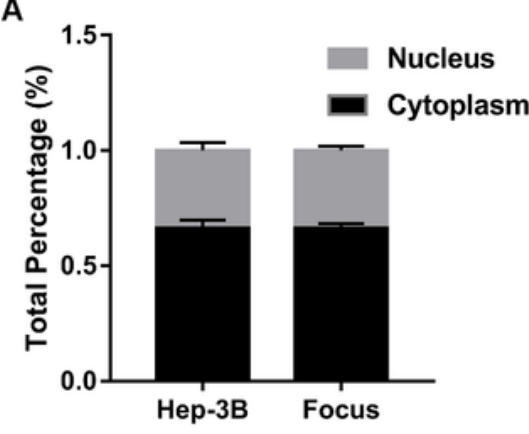

C

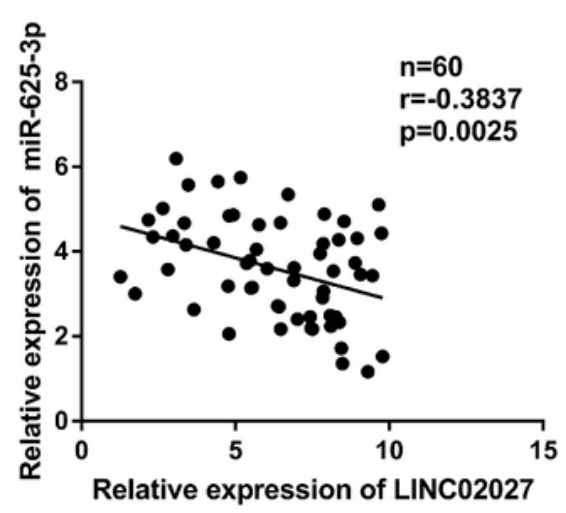

B

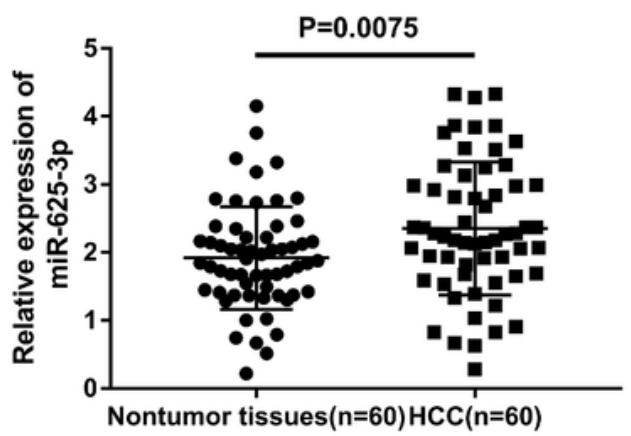

D

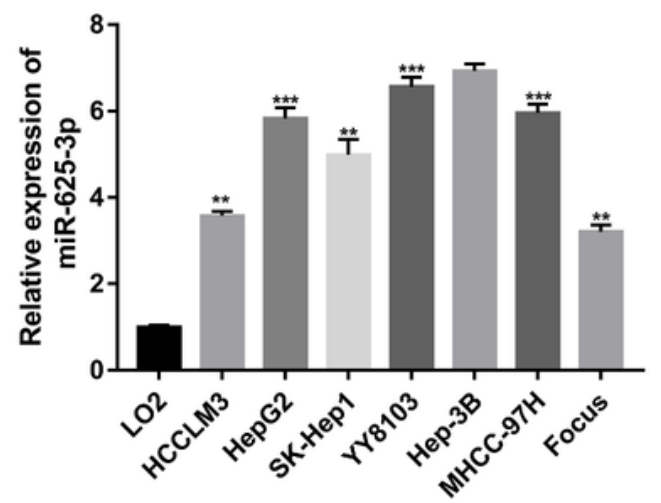

E

BindingSite chr3:81043039-81043060[+]

LINC02027-WT 5'...AUUAAGACCCUCAUCUAUAGUG... 3'

miR-625-3p 3'...ACUCCCCCUUUCAAGAUAUCAG... 5'

LINC02027-MUT 5'...AUUAAGACCCUCAAGAUAUCAG... 3'

$\mathbf{F}$
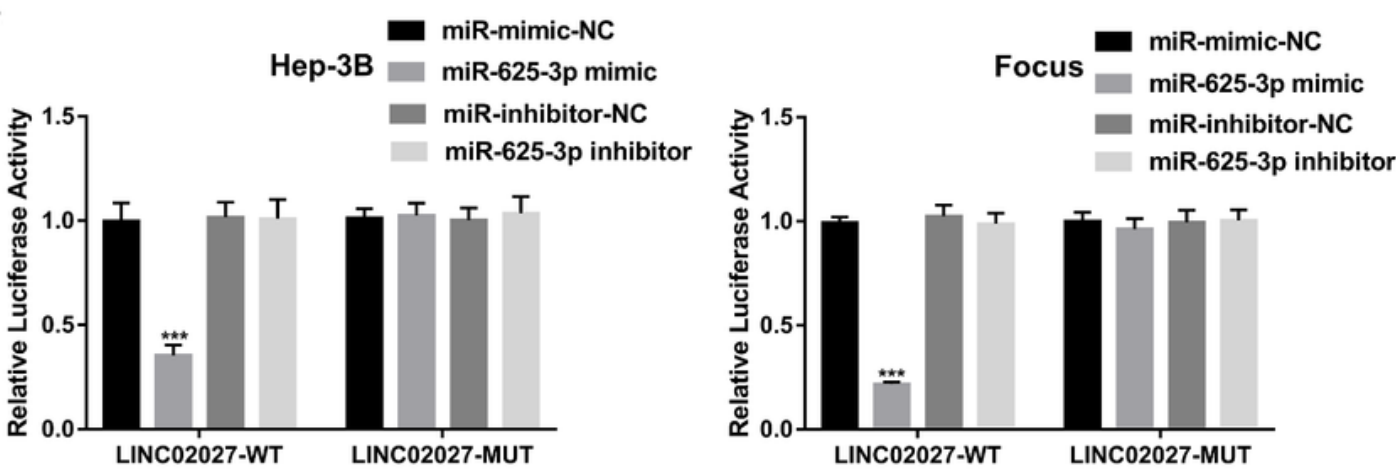


\section{Figure 4}

MiR-625-3p is the downstream regulator of LINC02027. A. Subcellular fractionation indicating that LINC02027 is located mainly in the cytoplasm. B. The expression of miR-625-3p was analyzed in 60 pairs of HCC tissues and related nontumor tissues. C. The analysis consequence of Spearman correlation analysis revealed the association between LINC02027 and miR-625-3p in the 60 HCC samples. D. The expression of miR-625-3p in HCC cell lines and immortalized human hepatocyte LO2. E. The predicted miR-625-3p targeting sequence with LINC02027. F. Luciferase activity was detected in luciferase reporter assay. The $p$ values represent comparisons between groups $\left({ }^{\star} p<0.05,{ }^{*} p<0.01,{ }^{\star *} p<0.001\right)$. 
A

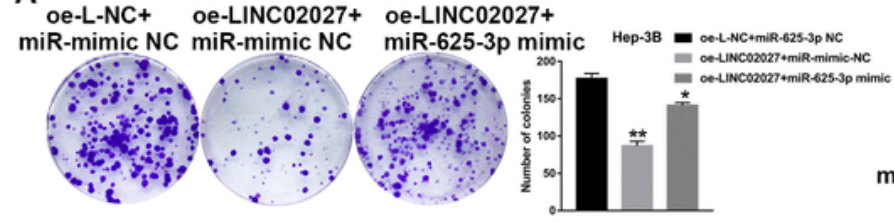

C

oe-L-NC+ oe-LINC02027+ oe-LINC02027+
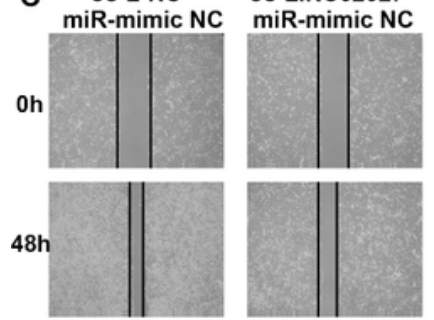

\section{miR-625-3p mimic}

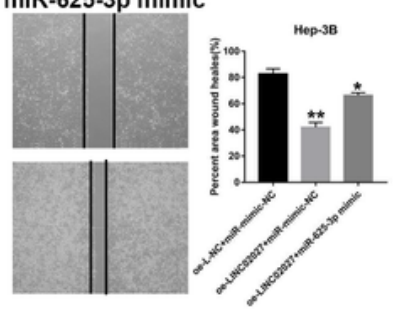

D

oe-L-NC+ oe-LINC02027+ oe-LINC02027+ miR-mimic NC miR-mimic NC miR-625-3p mimic

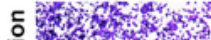

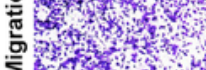

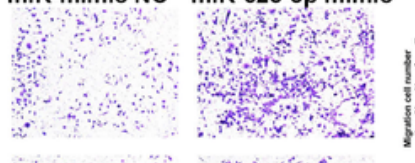

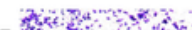

。盈榢

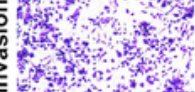

and
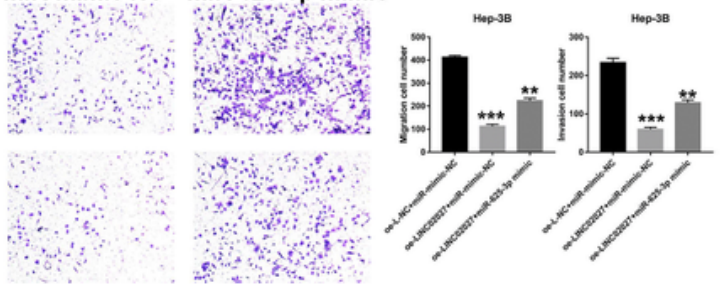

Vimentin

GAPDH

$\mathrm{Bcl}-2$

\section{.}

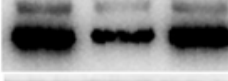

Bax

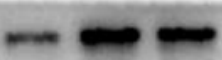

Caspase-3

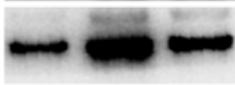

Caspase-9

E-cadherin

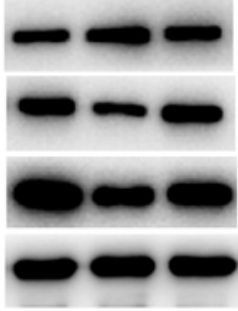

Hep-3B
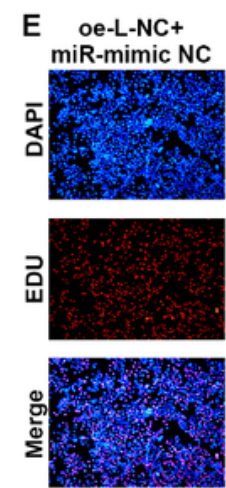

G
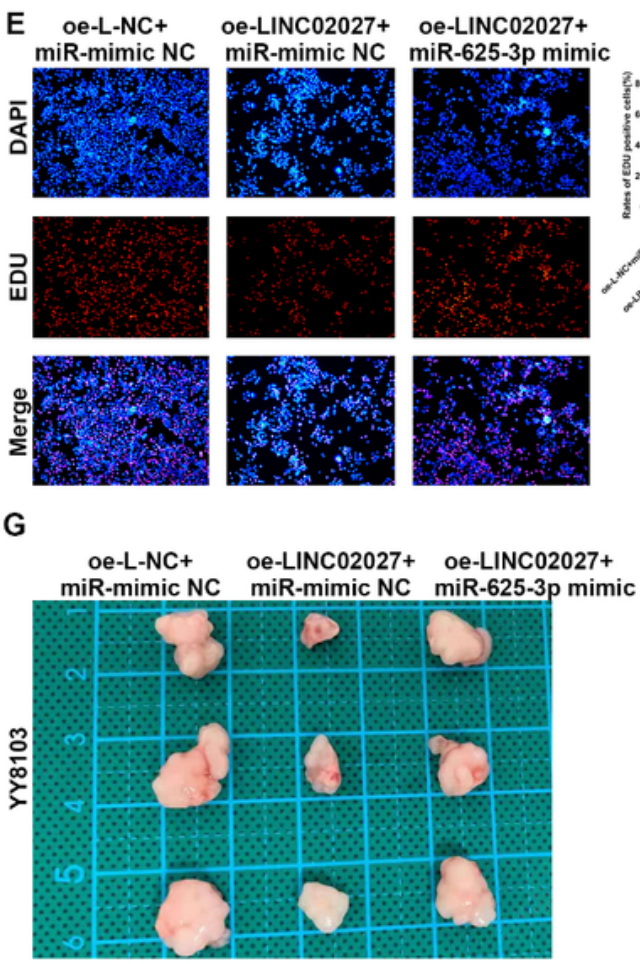
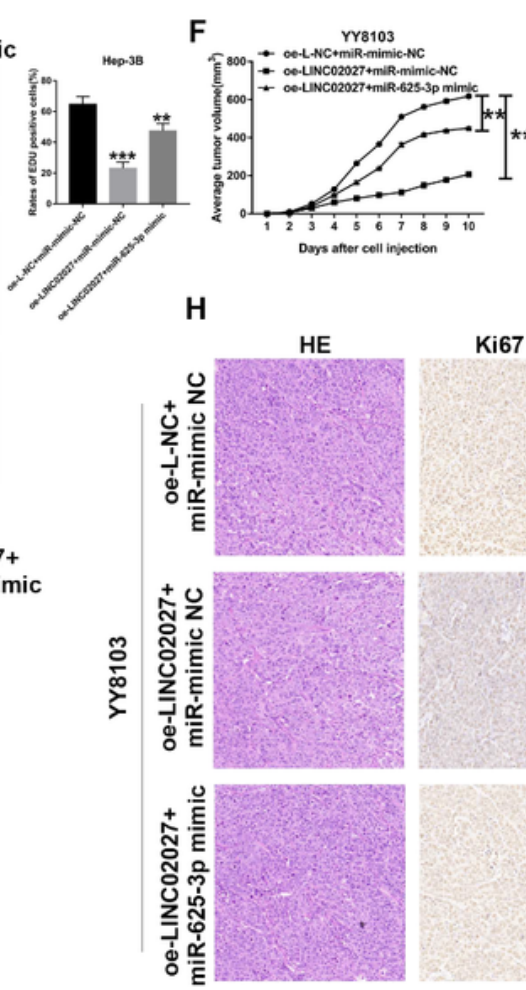

Ki67

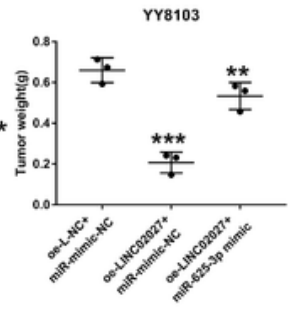

E-cadherin

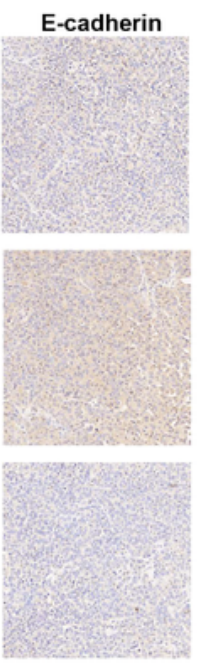

Figure 5

Rescue experiments were performed in vitro and vivo. A. The colony formation assay was performed to uncover colony forming ability of indicated groups. B. Bcl-2, Bax, Caspase-3, Caspase-9, E-Cadherin, NCadherin and Vimentin protein expression levels of the above progenitor cells. C. Wound healing assay was performed to evaluated the motility of indicated groups. D. Cell migration and invasion ability of HCC cells treated with diverse lentiviruses were evaluated by transwell assays. E. EDU test results of the target 
cell line. F. Tumor weight and volume were recorded and analyzed. G. Representative photographs of subcutaneous tumor tissues derived from indicated HCC cells. H. Representative images of HE staining and Immunohistochemical detection of subcutaneous tumor tissues $(x 400)$. ( ${ }^{*} P<0.05,{ }^{\star} P<0.01$, $* \star \star$ $\mathrm{P}<0.001)$.
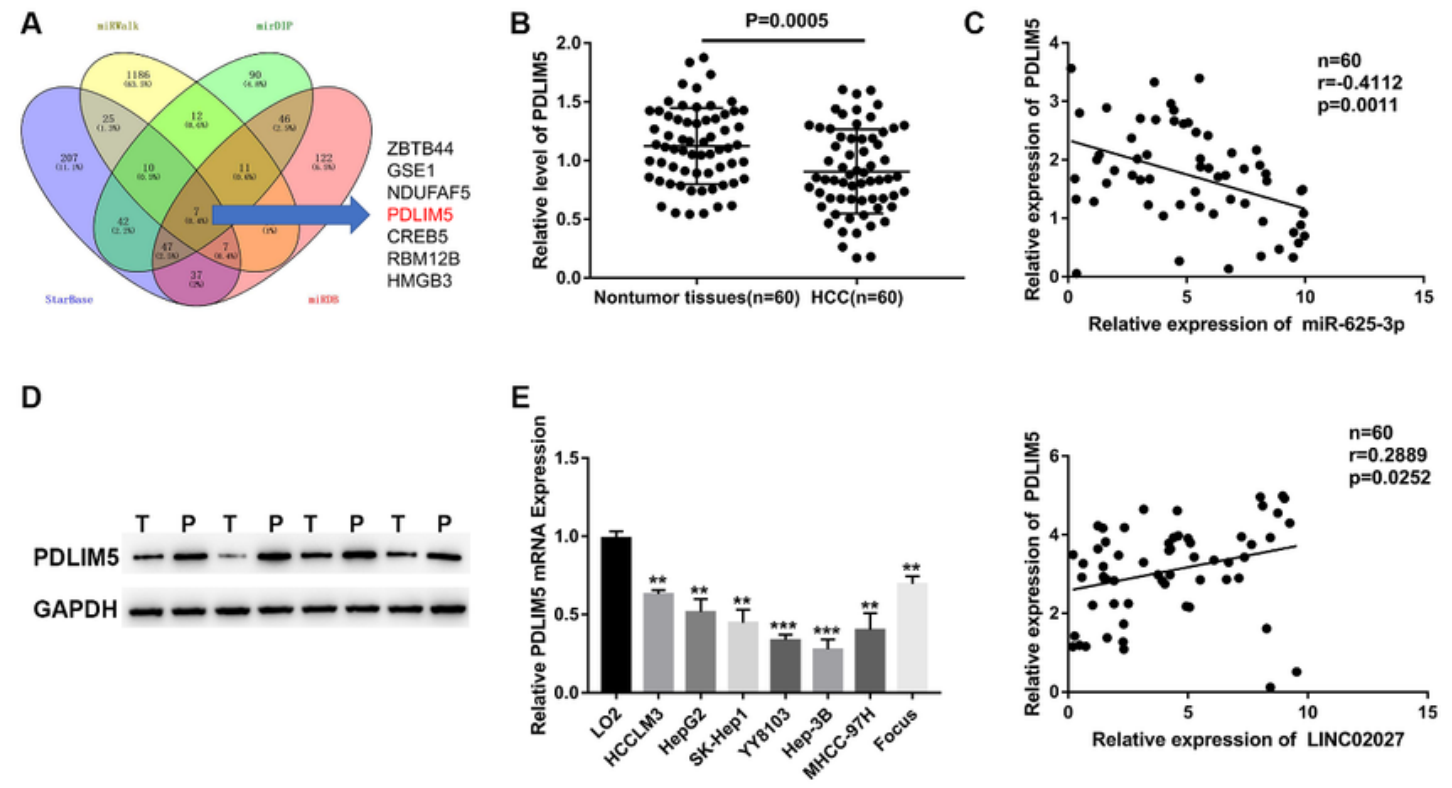

$\mathbf{F}$
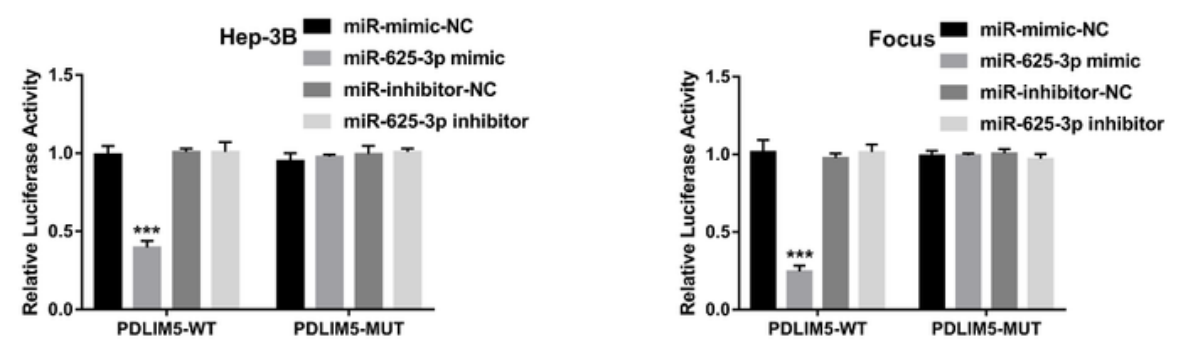

G

BindingSite chr4:95589315-95589320[+]
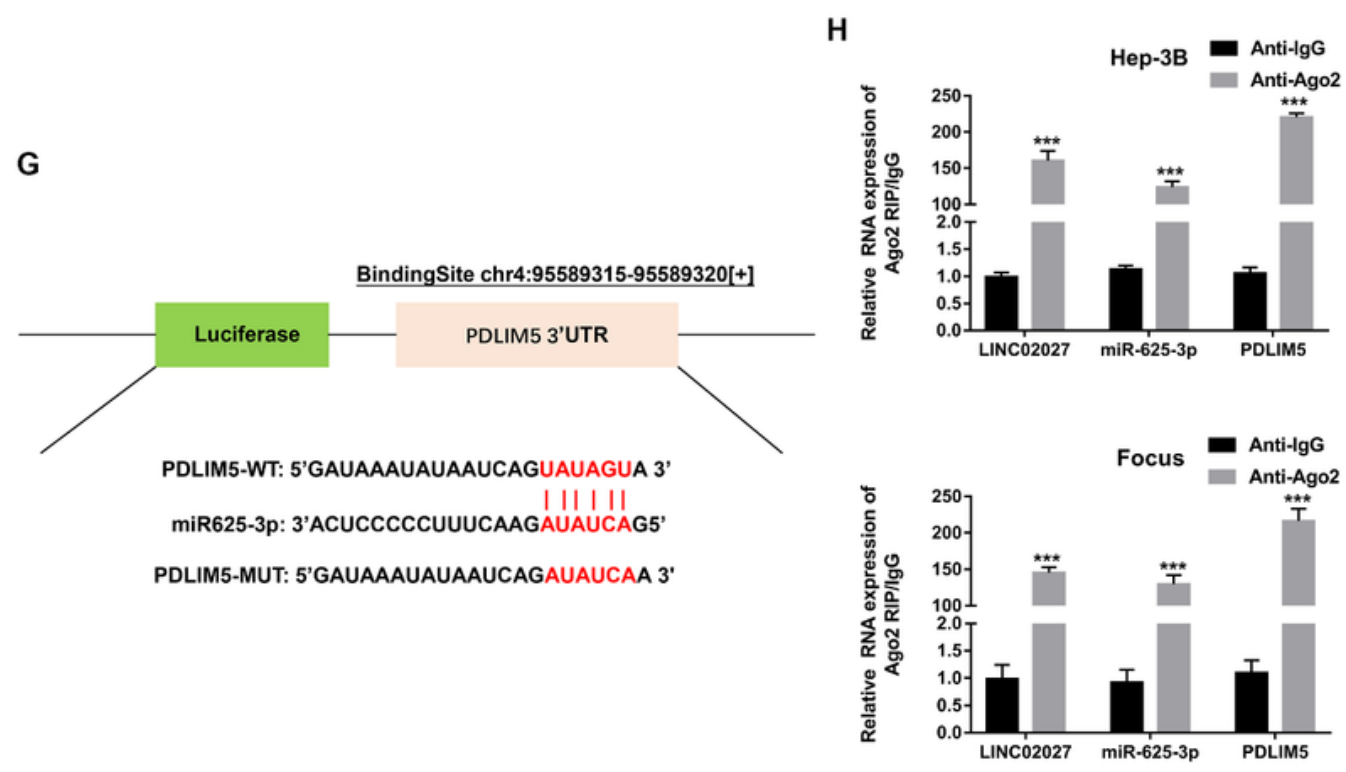

Figure 6 
PDLIM5 is the downstream target gene of miR-625-3p. A. Venn diagram showed that PDLIM5 was calculated and predicted as a downstream target gene of miR-625-3p by four prediction algorithms. B. The expression of PDLIM5 was analyzed in 60 pairs of HCC tissues and related nontumor tissues. C. The analysis consequence of Spearman correlation analysis revealed the association between LINC02027, miR-625-3p and PDLIM5 in the 60 HCC samples. D. Western blot assay was used to detect the amount of PDLIM5 in 4 pairs HCC tissues and related nontumor tissues. E. The expression of PDLIM5 in HCC cell lines and immortalized human hepatocyte LO2. F\&G. The predicted miR-625-3p targeting sequence with PDLIM5, and luciferase activity was detected in luciferase reporter assay. $\mathrm{H}$. RIP assay was used to detect the anti-AGO2 activity of LINC02027, miR-625-3p and PDLIM3 in 3B and Focus cell lines (with anti-IgG as control). (* $P<0.05,{ }^{* *} P<0.01$, *** $\left.P<0.001\right)$. 
A oe-L-NC+ oe-LINC02027+ oe-LINC02027+ sh-P-NC Sh-P-NC Sh-PDLIM5
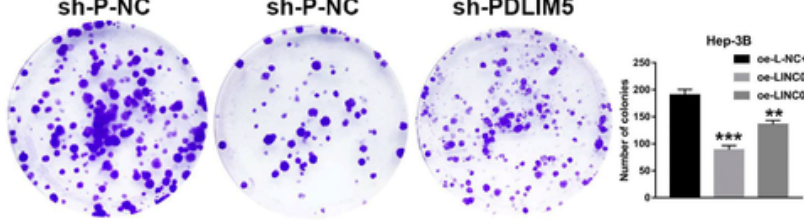

B

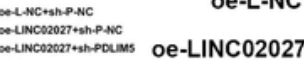

sh-P-NC

sh-PDLIM5


oe-LINC02027+

sh-PDLIM5
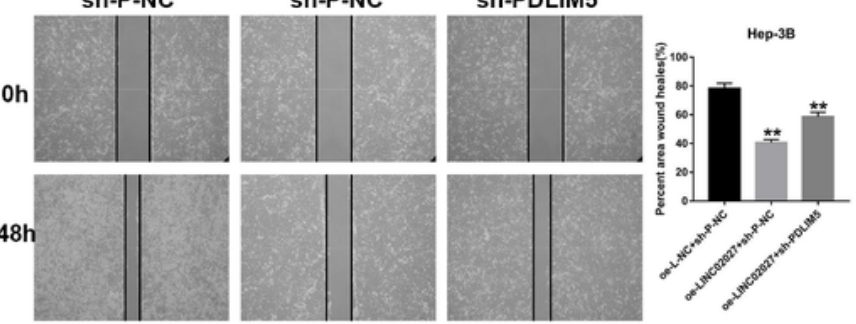

Raf

p-Raf

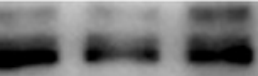

MEK

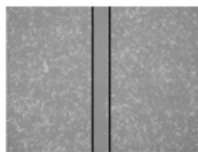

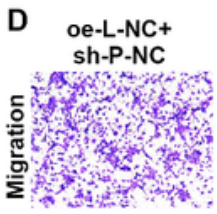
$\begin{array}{cc}\text { oe-LINC02027+ } \\ \text { Sh-P-NC } & \begin{array}{c}\text { oe-LINC02027+ } \\ \text { Sh-PDLIM5 }\end{array}\end{array}$
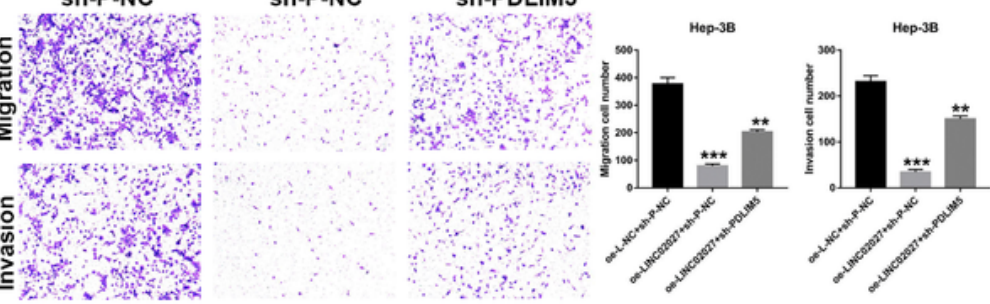

GAPDH

p-MEK

p-ERK

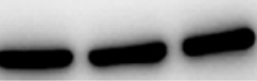

E

oe-L-NC+sh-P-NC oe-LINC02027+sh-P-NC oe-LINC02027+ sh-PDLIM5
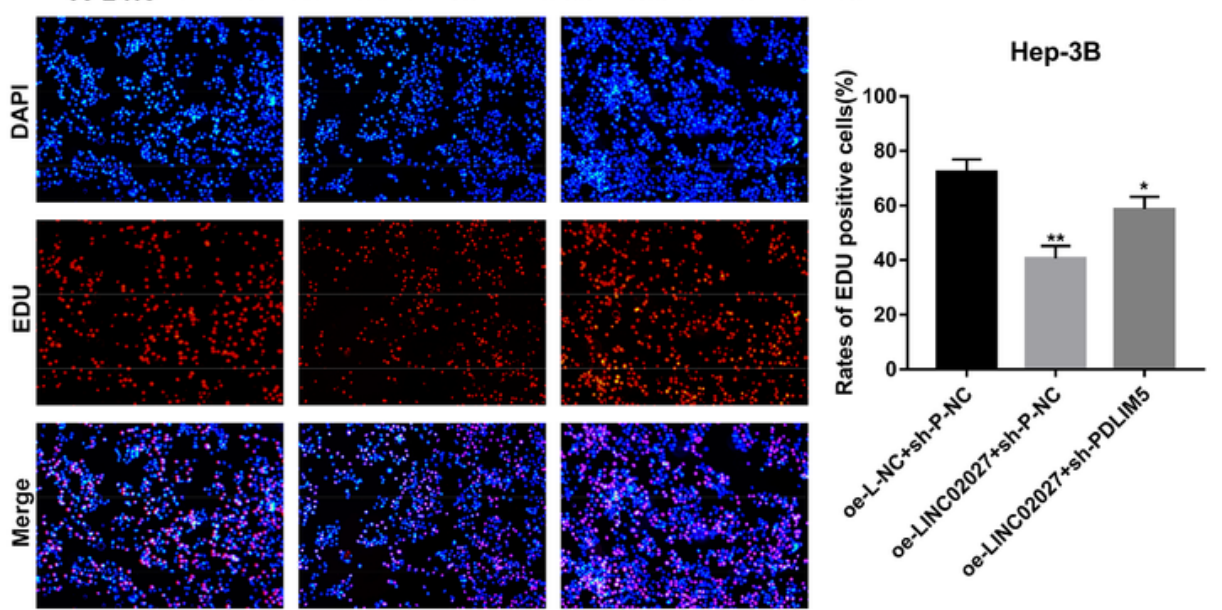

Figure 7

Rescue experiments were adopted to confirm that PDLIM5 is a downstream regulator of LINC02027. A. The colony formation assay was performed to uncover colony forming ability of indicated groups. B. LINC02027 and PDLIM5 regulate the RASA1 protein level and the expression levels of Raf, MEK, ERK and their related phosphorylated proteins in the RAS-MAPK pathway. C. Wound healing assay was performed 
to evaluated the motility of indicated groups. D. Cell migration and invasion ability of HCC cells treated with diverse lentiviruses were evaluated by transwell assays. E. EDU test results of the target cell line.
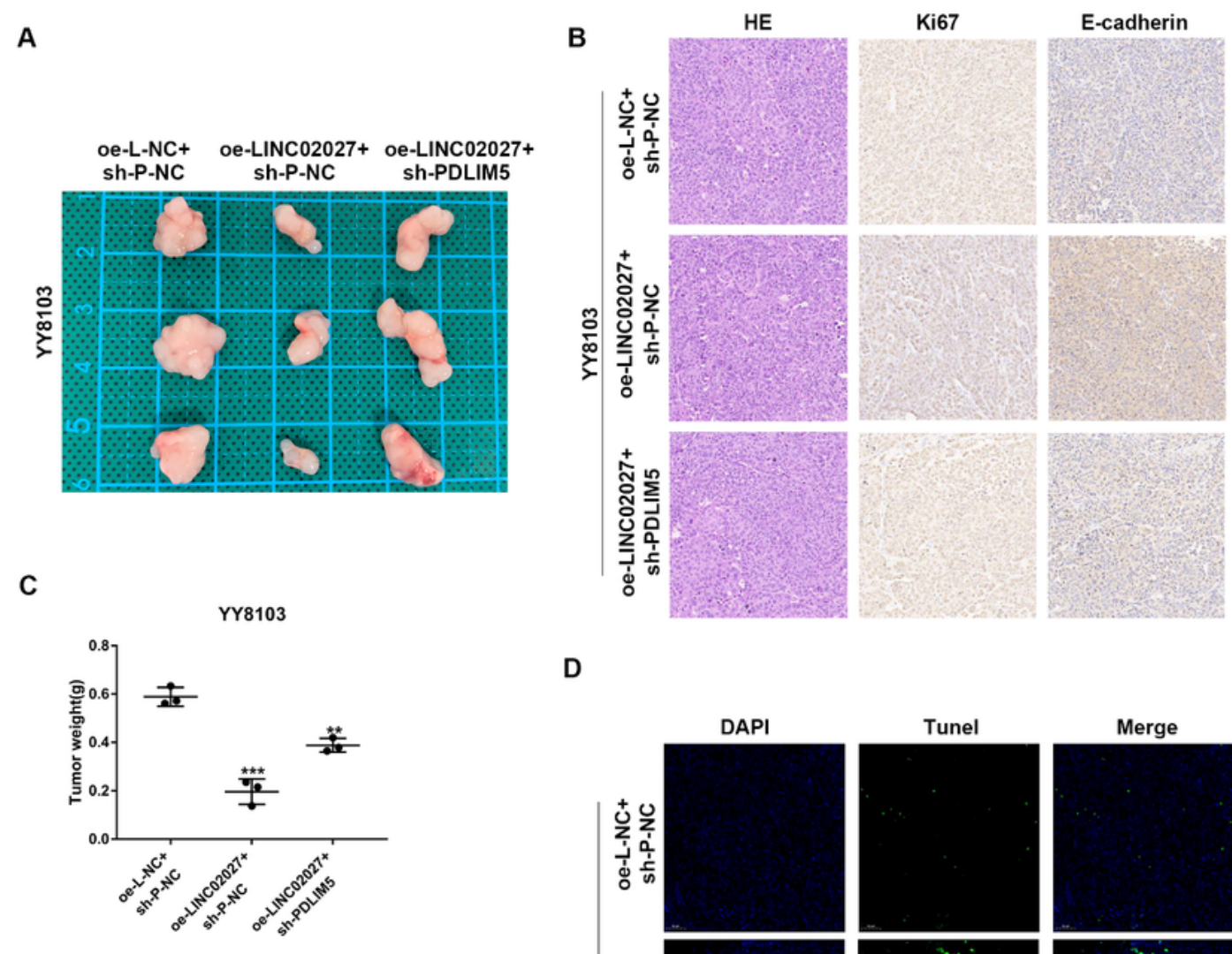

D
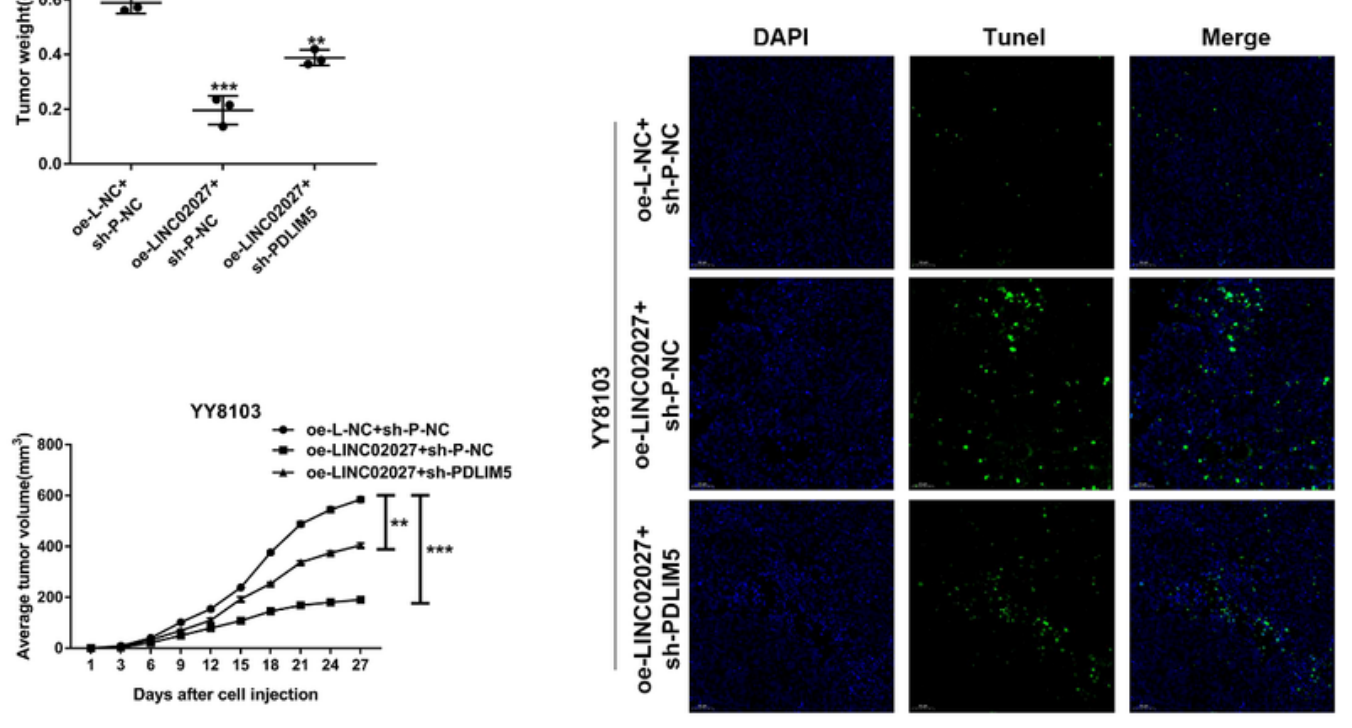

Figure 8

Rescue experiments were adopted in vivo. A. Representative photographs of subcutaneous tumor tissues derived from indicated HCC cells. B. Representative images of HE staining and Immunohistochemical 
detection of subcutaneous tumor tissues $(x 400)$. C. Tumor weight and volume were recorded and analyzed. D. Apoptosis was detected by TUNEL staining. ( $\left.{ }^{*}<0.05,{ }^{*} P<0.01, * \star * P<0.001\right)$.

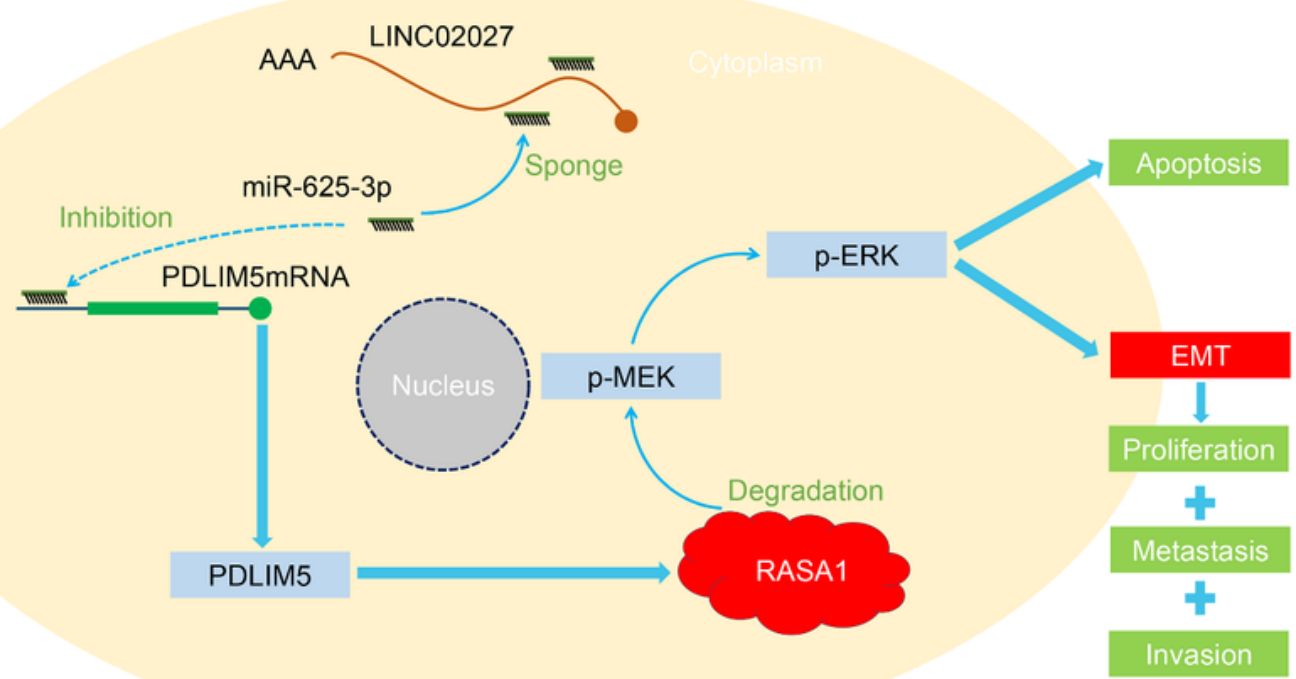

\section{Figure 9}

Graphical presentation of LINC02027/miR-625-3pPDLIM5/RAS/MAPK axis in HCC. LINC02027/miR-6253p/PDLIM5 Axis inhibits EMT and promotes apoptosis of HCC by regulating RAS-MAPK pathway. 


\section{Supplementary Files}

This is a list of supplementary files associated with this preprint. Click to download.

- Checklist.docx 